Z. klin. Chem. u. klin. Biochem.

7. Jg., S. 505-508, September 1969

\title{
Unveresterte freie Fettsäuren im Serum und agonales Kreislaufversagen
}

\author{
Von J. G. Gostomzyk und G. F. Frei \\ Aus dem Institut für gerichtliche Medizin und Versicherungsmedizin der Universität Freiburg i. Br. \\ (Direktor: Prof. Dr. W. Spann) \\ (Eingegangen am 19. Mai 1969)
}

Im Serum bis zu etwa 70 Stunden postmortal aus der V. femoralis und in einer Reihe von Fällen aus dem rechten Herzen entnommener Blutproben wurde die Konzentration der unveresterten freien Fettsäuren, der veresterten Fettsäuren und des Gesamtcholesterins bestimmt. In diesem Beobachtungszeitraum stieg die Konzentration der freien Fettsäuren im Serum in Abhängigkeit von der Todesursache unterschiedlich schnell an. Eine Konzentrationsänderung der veresterten Fettsäuren und des Gesamtcholesterins war nicht nachweisbar. Es wird ein Zusammenhang zwischen agonaler Katecholaminausschüttung und hohen Konzentrationen freier Fettsäuren bzw. hoher postmortaler lipolytischer Aktivität diskutiert. Offenbar führt die zeitliche Dissoziation zwischen Atemstillstand und Kreislaufstillstand zur Freisetzung lipolytischer Aktivität. Die Untersuchung agonaler und postmortaler Stoffwechselvorgänge erscheint uns wegen der Entwicklung der Transplantationschirurgie bedeutsam.

\section{The relationship between free serum fatty acids and agonal circulatory failure}

The concentration of free fatty acids, esterified fatty acids and total cholesterol was measured in serum from the V. femoralis and in blood samples from the right ventricle taken up to about 70 hours post mortem. During this period, the concentration of free fatty acids in the serum increased at various rates, depending on the cause of death. There was no detectable change in the concentration of esterified fatty acids and total cholesterol. The relationship between the agonal release of catecholamines, high free fatty acids concentrations and high post mortem lipolytic activity is discussed. Evidently the separation in time of respiratory and circulatory failure leads to the release of lipolytic activity. In view of the development of transplant surgery, the study of agonal and post mortem metabolism would seem to be important.

Nach akutem Myokardinfarkt ist die Konzentration der unveresterten freien Fettsäuren im Serum erhöht (1). Dabei bestehen offenbar Beziehungen zwischen der Konzentrationserhöhung freier Fettsäuren nach einem Infarkt und dem Auftreten von Arrhythmien und plötzlichem Tod (2).

Von den Substanzen mit lipolytischen Eigenschaften wie Noradrenalin, Adrenalin, Glucagon, Glucocorticoide, ACTH, TSH, STH, Vasopressin und einigen hypophysären Polypeptiden dürfte den kreislaufaktiven Katecholaminen nach dem Infarktereignis und für agonal bzw. postmortal nachweisbare Erhöhungen des freie Fettsäuren-Spiegels eine besondere Bedeutung zukommen. Es wurde deshalb untersucht, ob sich durch Bestimmung der freien Fettsäuren in postmortal entnommenen Blutproben Unterschiede im Kreislaufversagen erkennen lassen.

\section{Methoden}

Zur Untersuchung wurden Blutproben zu verschiedenen Zeiten nach Eintritt des Todes aus der V. femoralis entnommen. In einer Reihe von Fällen wurde weiterhin während der Obduktion Blut aus dem rechten Herzen und der V. cava durch Einschneiden in die V. cava unmittelbar über der Einmündung in den rechten Vorhof gewonnen. Die Blutproben wurden zentrifugiert und das teilweise hämolytische Serum bis zur Untersuchung eingefroren. Die Feststellung der Todesursache erfolgte durch die Obduktion. Ein Diabetes mellitus war in keinem der Fälle bekannt. Die Bestimmung der freien Fettsäuren exfolgte titrimetrisch nach der $\mathrm{Me}$ thode von Dole in der Modifikation von Trout (3). Weiterhin wurde die Konzentration der veresterten Fettsäuren kolorimetrisch nach Fried und Hogfumayer (4) sowie die des Gesamtcholesterins photometrisch in einer Modifikation der Liebermann-BurchardReaktion $(5,6)$ bestimmt.
Die Leichen wurden im Institut bei $4^{\circ}$ gelagert. Die Lagerungszeit in Umgebungstemperatur bis zur Einlieferung in das Institut betrug für: Gruppe I 3,7 \pm 10,0 Stdn, Gruppe II 9,7 \pm 6,3 Stdn, Gruppe III $7 \pm 12,8$ Stdn, Gruppe IV 18,1 $\pm 12,1$ Stdn, Gruppe V 7,7 $\pm 5,9$ Stdn.

\section{Ergebnisse}

In den untersuchten Serumproben, die teilweise wenige Stunden nach dem Tode entnommen worden waren, wurden verschieden hohe Konzentrationen freier Fettsäuren gefunden. Postmortal steigen die Konzentrationen freier Fettsäuren an, wobei die lipolytische Aktivität im Blut bei verschiedenen Todesursachen unterschiedlich groß ist. Der postmortale Anstieg der Konzentrationen freier Fettsäuren, wie er sich in Abbildung 1 aus den Einzelbestimmungen ergibt, wurde in einer Reihe von Fällen durch 2 Blutentnahmen an derselben Leiche zu verschiedenen Zeitpunkten bestätigt.

Nach der Höhe der postmortal nachgewiesenen Konzentrationen freier Fettsäuren, die von der vor dem Tode herrschenden Stoffwechselsituation, der agonalen Kreislaufregulation und der postmortalen lipolytischen Aktivität abhängt, läßt sich das Untersuchungsgut unter Berücksichtigung der Todesursache in folgende Gruppen einteilen:

A. Normale Konzentrationen freier Fettsäuren kurze Zeit nach Eintritt des Todes und geringe postmortale lipolytische Aktivität:

Gruppe I: Perakuter Unfalltod durch Schädelhirntrauma $(n=10)$, Ruptur großer Gefäße $(n=4)$.

Gruppe II: Tod durch Kreislaufversagen mit langsamer Agonie $(n=34)$, z. B. nach Pneumonie, Tablettenvergiftung, Nierenversagen, Peritonitis. 


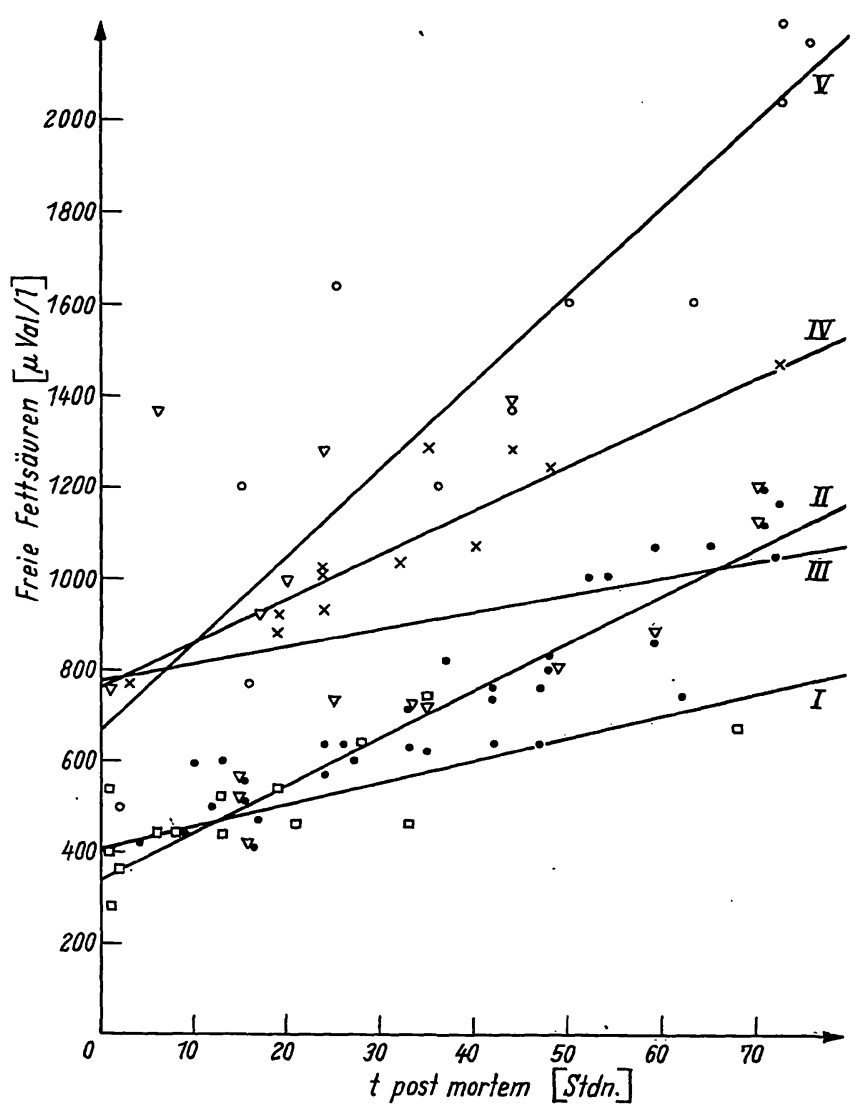

Abb. 1

Die Konzentrationen der unveresterten freien Fettsäuren (FFS) wurden in Abhängigkeit vom Zeitpunkt der Blutentnahme aus der V. femoralis post mortem aufgetragen. Nach ihrer Todesursache wurden die untersuchten Fälle 5 Gruppen zugeordnet $(I=\square=$ akuter Unfalltod durch Schädelhirntrauma, II $=\cdot$ = Herz- und Kreislaufversagen mit langsamer Agonie, III $=\Delta=$ akutes Herzversagen mit kurzer Agonie IV $=\times=$ Ersticken bzw. akuter Hirndruck,
$\mathrm{V}=0=$ Erhängen) stimmungen wurde für jede Gruppe die Regressionsgerade berechnet Der Anstieg der Regressionsgerade ist Ausdruck postmortaler lipolyDer Anstieg der Regressionsgerade ist Ausdru Regressionsgeraden

I $\mathrm{y}=408+4,9 \mathrm{x} \quad$ II $\mathrm{y}=338+10,3 \mathrm{x} \quad$ III $\mathrm{y}=776+3,74 \mathrm{x}$ IV $y=760+9,69 x \quad$ V $y=666+19,04 x$

B. Normale bzw. erhöhte Konzentrationen freier Fettsäuren kurze Zeit nach Eintritt des Todes und hohe postmortale lipolytische Aktivität:

Gruppe III: Akutes Herzversagen mit kurzer Agonie $(n=17)$, wobei der Tod bereits vor dem Eintreffen in die Klinik eingetreten war.

Gruppe IV: Deutlich erhöhte Konzentrationen freier Fettsäuren wurden weiterhin bei folgenden Todesur- sachen gefunden: Ertrinken (1 Fall), Ersticken durch Aspiration (3 Fälle), CO-Vergiftung (1 Fall), Ersticken durch Thoraxkomprèssion (1 Fall), Stromtod (1 Fall), intrakranielle Blutung (1 Fall) und Kopfschuß (5 Fälle). Es handelt sich also um Fälle von Ersticken oder plötzlichem intrakraniellem Druckanstieg, der möglicherweise innerhalb kurzer Zeit zur zentralen Atemlähmung führte.

Gruppe V: Diese Gruppe umfaßt Personen, die Suicid durch Erhängen vorgenommen hatten ( $n=11$ ).

Die festgestellten Konzentrationen freier Fettsäuren sind in Abbildung 1 in Abhängigkeit vom Zeitpunkt der Blutentnahme nach Eintritt des Todes angegeben. Aus den Meßpunkten läßt sich für jede der angegebenen Diagnosegruppen I- $\mathrm{V}$ eine Regressionsgerade berechnen, deren Steigung Ausdruck der unterschiedlich hohen postmortalen lipolytischen Aktivität ist.

Ein signifikanter postmortaler Anstieg der Konzentrationen der veresterten Fettsäuren und des Gesamtcholesterins im Serum ließ sich während des Beobachtungszeitraumes nicht nachweisen.

In der Tabelle 1 sind die Mittelwerte der Konzentrationen der freien Fettsäuren, der unveresterten Fettsäuren und des Gesamtcholesterins angegeben. Diese Lipoide weisen innerhalb der gebildeten Gruppen statistisch signifikante Unterschiede auf. Bleibt das unterschiedliche mittlere Lebensalter der jeweiligen Gruppen unberücksichtigt, so war in der Gruppe der an ankutem Herztod Verstorbenen, verglichen (T-Test nach STUDENT) mit dem an Unfallfolgen perakut Verstorbenen, der Serumgehalt an freien Fettsäuren $(t=4,83$, $p$ $<0,001)$, an veresterten Fettsäuren $(t=5,05$, $p$ $<0,001)$ und an Gesamtcholesterin $(t=8,35$, $p<0,001)$ signifikant erhöht.

Beim statistischen Vergleich ( $t$-Test) der entsprechenden Werte (Tab. 1) zwischen den an Strangulation und den den nach Unfällen mit Schädelhirntrauma Verstorbenen zeigte die erstgenannte Gruppe signifikant höhere Werte für freie Fettsäuren $(t=6,54, p<0,001)$, für veresterte Fettsäuren $(t=3,75, p<0,005)$ und für Gesamtcholesterin $(t=2,99, p<0,01)$.

Der Gruppenvergleich der Werte ( $t$-Test) zwischen den an akutem Herztod und an Strangulation Verstorbenen (Tab. 1) ergibt, daß die Konzentrationen freier Fettsäuren bei den Erhangenen signifikant höher liegen $(t=3,63$,

Tab. 1

Für einzelne Gruppen mit einheitlicher Todesursache wurde die mittlere Konzentration der unveresterten freien Fettsäuren (FFS), der veresterten Fettsäuren und des Gesamtcholesterins berechnet. Neben der mittleren Entnahmezeit der Blutproben post mortem aus der V. femoralis werden das durchschnittliche Lebensalter und das Körpergewicht mit einfacher Streuung angegeben

\begin{tabular}{|c|c|c|c|}
\hline \multirow{2}{*}{$\begin{array}{c}\text { Diagnose } \\
\text { akuter Unfalltod (Schädel-Hirn-Trauma) } \\
48,4 \pm 20,2 \text { Jahre } \\
66,2 \pm 8,1 \mathrm{~kg} \mathrm{KG}\end{array}$} & \multirow{2}{*}{$\begin{array}{l}\text { Blutentnahme } \\
\text { Stdn. p. m. } \\
17,8 \pm 18,7 \\
21,3 \pm 26,6 \\
22,0 \pm 27,4\end{array}$} & \multicolumn{2}{|c|}{ Plasmakonzentrationen (V. femorlis) } \\
\hline & & $\begin{array}{c}\text { FFS }(n=14) \\
\text { veresterte Fetts. }(n=14) \\
\text { Cholesterin }(n=12)\end{array}$ & $\begin{array}{l}493 \pm 136 \mu \mathrm{Val} / \mathrm{l} \\
305 \text { 圭 } 85 \mathrm{mg} / 100 \mathrm{ml} \\
200 \mathrm{I} 3 \mathrm{mg} / 100 \mathrm{ml}\end{array}$ \\
\hline $\begin{array}{c}\text { akuter Herztod } \\
58,3 \pm 15,3 \text { Jahre } \\
70,9 \pm 11,5 \mathrm{~kg} \mathrm{KG}\end{array}$ & $\begin{array}{l}34,3 \pm 24,8 \\
30,5 \pm 27,5 \\
30,3 \pm 21,4\end{array}$ & $\begin{array}{l}\text { FFS }(n=17) \\
\text { veresterte Fetts. }(n=15) \\
\text { Cholesterin }(n=14)\end{array}$ & $\begin{array}{l}905 \pm 290 \mu \mathrm{Val} / \mathrm{l} \\
559 \pm 171 \mathrm{mg} / 100 \mathrm{ml} \\
305 \pm 38 \mathrm{mg} / 100 \mathrm{ml}\end{array}$ \\
\hline $\begin{array}{c}\text { Strangulation } \\
36,7 \pm 14,9 \text { Jahre } \\
72,9 \pm 12,0 \mathrm{~kg} \mathrm{KG}\end{array}$ & $\begin{array}{l}42,7 \pm 25,3 \\
39,5 \pm 24,9 \\
39,5 \pm 24,9\end{array}$ & $\begin{array}{l}\text { FFS }(n=11) \\
\text { veresterte Fetts. }(n=10) \\
\text { Cholesterin }\end{array}$ & $\begin{aligned} 1479 & \pm 545 \mu \mathrm{MVal} / \mathrm{l} \\
586 & \pm 265 \mathrm{mg} / 100 \mathrm{ml} \\
303 & \pm 125 \mathrm{mg} / 100 \mathrm{ml}\end{aligned}$ \\
\hline
\end{tabular}


$\mathrm{p}<0,005)$, dagegen bestehen für veresterte Fettsäuren $(t=0,295, \quad p<0,80)$ und für Gesamtcholesterin $(t=0,056, p<1,0)$ keine statistisch signifikanten Unterschiede.

In einer Reihe von Fällen wurden die Bestimmungen der freien Fettsäuren, der veresterten Fettsäuren und des Gesamtcholesterins neben der Untersuchung im Serum aus Blut der Oberschenkelvene auch in den aus dem rechten Herzen und der V. cava entnommenen Proben durchgeführt. Die Konzentrationen der freien Fettsäuren lagen hierbei in allen Fällen in dem aus dem letztgenannten Stromgebiet entnommenen Proben höher als im Blut der V. femoralis. Wie in Tabelle 2 angegeben,

Tab. 2

Angegeben sind die Differenzen zwischen den Lipoidkonzentrationen im Blut des rechten Herzens und der $V$. cava zur Konzentration im Blut der V. femoralis. Die Konzentrationen der unveresterten freien Fettsäuren (FFS) im Blut aus dem rechten Herzen liegen höher als im Blut aus der V. femoralis, die Konzentrationen der veresterten Fett-
săuren und des Gesamtcholesterins sind in beiden Gefäßabschnitten praktisch gleich

\begin{tabular}{|c|c|c|c|}
\hline \multirow[b]{2}{*}{ Diagnosen } & \multicolumn{3}{|c|}{$\begin{array}{c}\text { Konzentrationsdifferenzen re Herz - } \\
\text { v. femoralis }\end{array}$} \\
\hline & $\begin{array}{c}\text { FFS } \\
\mu \mathrm{Val} / l\end{array}$ & $\begin{array}{c}\text { veresterte } \\
\text { Fettsäuren } \\
\mathrm{mg} / 100 \mathrm{ml}\end{array}$ & $\begin{array}{l}\text { Cholesterin } \\
\mathrm{mg} / 100 \mathrm{ml}\end{array}$ \\
\hline $\begin{array}{l}\text { akuter Unfalltod } \\
\text { (Schädel-Hirn- } \\
\text { Trauma }\end{array}$ & $\begin{aligned} 393 & \pm 229 \\
(\mathrm{~N} & =4)\end{aligned}$ & $\begin{array}{l}-26 \pm 42 \\
(N=4)\end{array}$ & $\overline{(N}^{5} \pm 53$ \\
\hline akuter Herztod & $\begin{aligned} 246 & \pm 193 \\
(N & =5)\end{aligned}$ & $\begin{array}{l}23 \pm 35 \\
(\mathrm{~N}=6)\end{array}$ & $\begin{aligned} 16 & \pm 38 \\
N & =5\end{aligned}$ \\
\hline Strangulation & $\begin{array}{c}729 \pm 447 \\
(\mathrm{~N}=8)\end{array}$ & $\left(N^{7} \pm 75\right)$ & $\begin{array}{c}-24 \pm 84 \\
N\end{array}$ \\
\hline
\end{tabular}

ist die Höhe der Konzentrationsdifferenz unterschiedlich bei verschiedenen Todesursachen. Beim statistischen Vergleich (t-Test Paaranalyse) hatten Personen nach perakutem Unfalltod signifikant größere Differenzen als Personen, bei denen akutes Herzversagen die Todesursache war $(t=4,91, p<0,005)$. Die Differenz war in der letztgenannten Gruppe signifikant kleiner als bei Strangulierten $(t=7,69, p<0,001)$. Auch gegenüber der Gruppe der Unfalltoten wiesen die Strangulierten signifikant höhere Konzentrationsdifferenzen auf $(t=4,73$, $\mathrm{p}<0,001)$.

\section{Diskussion}

Die agonale Stoffwechselsituation, ihre Steuerung, sowie postmortale biochemische Befunde. haben durch die Entwicklung der Transplantationschirurgie besonderes Interesse erlangt.

Im Fettstoffwechsel kommt der Fraktion der freien Fettsäuren im Serum eine zentrale Stellung zu. Für ihre Freisetzung im Fettgewebe im Zuge der Lipolyse kann zwischen einer sympathico-adrenergischen und einer nichtadrenergischen Aktivierung der Lipolyse unterschieden werden. Die u. a. durch die biogenen Amine aktivierbare „hormonsensitive“ Triglycerid-Lipase ist verschieden von den durch die in der Einleitung genannten Substanzen mit lipolytischen Eigenschaften nicht aktivierbarem Enzymsystem der "Monoglycerid-Lipasen“ (7).
Offenbar aktivieren die Katecholamine zunächst die Adenyl-Cyclase. Die Lipaseaktivierung erfolgt dann durch Adenosin-3', 5'-monophosphat, das unter dem Einfluß der Adenyl-Cyclase aus ATP gebildet wird (8). In vivo führt die Injektion von Noradrenalin $(9,10,11)$ und auch von Adrenalin (12) zu einem signifikanten Anstieg der Konzentration freier Fettsäuren im Blut. Werden $0,25 \mu \mathrm{g} / \mathrm{Min} /$ pro kg Körpergewicht beim Gesunden injiziert, so steigt die Konzentration freier Fettsäuren innerhalb der ersten Stunde um 100,6 $\pm 49,25 \%$ des Ausgangswertes an (12). In vitro führt die Inkubation von Fettgewebe mit Noradrenalin zur Freisetzung unveresterter Fettsäuren $(13,14)$.

Die Ausschüttung von Katecholaminen im Organismus ist vom Sauerstoffgehalt des Blutes abhängig. Mit abnehmender $\mathrm{O}_{2}$-Sättigung steigt der Adrenalin-Spiegel im Blut (15). Entsprechend wurden postmortal im Blut Erhängter höhere Katecholamin-Konzentrationen gefunden als bei akutem Tod durch gewaltsame Ursache (16). Die postmortalen Katecholamin-Spiegel im Serum waren nach Sekundenherztod höher als nach langsamem Herzversagen mit Agonie über Stunden (16). Weiter führte im Tierexperiment ein schneller intrakranieller Druckanstieg zur Ausschüttung von Katecholaminen, dagegen war ein langsamer Druckanstieg diesbezüglich unwirksam (16).

In den vorliegenden Untersuchungen wurden die höchsten Konzentrationen freier Fettsäuren bzw. die stärkste lipolytische Aktivität nach Erhängen (Gruppe V) oder Ersticken (Teil der Gruppe IV) beobachtet. Für die übrigen Fälle der Gruppe IV (Kopfschuß, akuter Tod nach Hirnblutung) erscheint die Annahme berechtigt, daß der intrakranielle Druckanstieg schnell zu einer zentralen Atemlähmung führte. Danach hat in diesen Fällen der Kreislauf die Atmung eine Zeitlang überlebt, so daß hier die $\mathrm{O}_{2}$-Verarmung des Blutes als Reiz für eine Kätecholaminausschüttung wahrscheinlich ist.

Beim akuten Herzversagen kommt der plötzliche Blutdruckabfall und der dadurch hervorgerufene $\mathrm{O}_{2}$-Mangel als auslösender Reiz für die Katecholaminausschüttung und den folgenden Anstieg freier Fettsäuren in Betracht. Daraus ergeben sich Diskussionspunkte für die Klärung des Zusammenhanges zwischen Myokardinfarkt und dem Auftreten von Kammerflimmern und plötzlichem Herzstillstand. Es kann ein direkter oder aber ein relativer Sauerstoffmangel, entstanden durch das hohe Angebot an freien Fettsäuren als Energiesubstrat mit hohem $\mathrm{O}_{2}$-Verbrauch im Stoffwechsel an den Herzmuskel, ursächlich sein (2).

Der postmortal zu beobachtende Anstieg der Konzentrationen freier Fettsäuren deutet auf das Vorhandensein lipolytischer Aktivität im Blut hin, wobei diese im Blut der V. cava und des rechten Herzens höher als im Blut der V. femoralis ist. Offenbar führt der Sauerstoffmangel zum Ubertritt von Lipasen aus hypoxisch geschädigten Zellen, insbesondere aus den Leberzellen, in die Blutbahn. Die Menge der aus den Zellen austretenden Lipasen wird dabei möglicherweise von der Zeitdauer zwischen Atemstillstand und Kreislaufstillstand beeinflußt. Gegen eine 
direkte Aktivierung von Lipasen in der Leber durch Katecholamine spricht die beobachtete kleine Differenz zwischen den Konzentrationen freier Fettsäuren im Blut der V. cava und der V. femoralis nach plötzlichem Herztod, obwohl nach Myokardinfarkt im Harn (17) und nach akutem Herztod im Blut (16) hohe Katecholaminkonzentrationen nachgewiesen wurden.
Nach den Ergebnissen der bisherigen Untersuchungen gehen unsere Vorstellungen dahin, daß postmortal hohe Konzentrationen freler Fettsäuren bzw. eine hohe lipolytische Aktivität im Blut indirekt auf eine agonale $\mathrm{Ka}$ techolaminausschüttung und weiterhin auf eine zeitliche Dissoziation zwischen Atemstillstand und Kreislaufstillstand hinweisen, wobei letzterer jeweils später erfolgt ist.

\section{Literatur}

1. KuRIen, V. A. und M. F. Oliver, Lancet London 1966/II, 122. 2. Oliver, M. F., V. A. Kurien und T. W. Greenwood, Lancet London, 1968/I, 710. - 3. ZöLLNER, N. und D. EBERHAGEN, Untersuchungen und Bestimmung der Lipoide im Blut, S. 339, Springer, Berlin-Heidelberg-New York (1965). 4. Fried, R. und J. Hoeflmayer, Klin. Wschr. 41, 727 (1963). 5. Liebermann, C., Ber. Dtsch. Chem. Ges. 18, 1803 (1885); H. Burchard, Inaug. Diss. Rostock (1889). - 6. Pearson, S., S. Stern und T. H. McGavack, Analytic Chem. 25, 813 (1953). 7. Böhle E., H. Ditschunert, F. Melani, J. Beyer, K. SchöfFIING und E. F. PFeiffer, Dtsch. med. Wschr. 91, 1083 (1966). 8. BRECH, W. J. und E. S. Gordon, Klin. Wschr. 45, 906 (1967). 9. Gordon, R. S. und A. Cherkes, J. clin. Invest. 35, 206 (1956), 10. Eggstein, M., W. KNOdel, H. KRämer und P. Baettaner,
Klin. Wschr. 45, 943 (1967). - 11. Fredrickson, D. S. und R. S. Gordon, Physiol. Rev. 38, 585 (1958). - 12. SANDhofer, F., S. SAILER, F. Dienste, H. BRAunsteiner, Klin. Wschr. 45, 486 (1967). - 13. Whrte, J. E. und F. I. Enger, Proc. Soc. exp. Biol. (N. Y.), 99, 375 (1958). - 14. VaughaN, M. und D. STerNBERG, Handbook of Physiology, sect. 5, p. 239, Waverly Press, Inc., Baltimore (1965). - 15. LudemanN H. A., M. G. FilberT und M. Corndilath, J. appl. Physiol. 8, 59 (1955). - 16. Laves, W. und S. Berg, Agonie, Physiologisch-chemische Untersuchungen bei gewaltsamen Todesarten. Verlag Max-Schmidt-Römhild, Lübeck (1965). - 17. WALLACE A. G., In Acute Myocardial Infarction, D. G. Julian und M. F. Oliver, Edinburgh zitiert nach M. F. Oliver, V. A. Kurien und T. W. Greenwood, Lancet London 1968/I, 710.
Dr. J. Gostomzyk 78 Freiburg i. Br. Albertstraße 9 